\title{
Passive Obstacle Detection System (PODS) for Wire Detection
}

\author{
John N. Sanders-Reed, Dennis J. Yelton, Christian C. Witt, Ralph R. Galetti*
}

\begin{abstract}
Boeing has developed algorithms and processing to detect power lines and cables in passive imagery from a wide variety of different sources. The algorithm has been demonstrated with imagery from visible, medium and long wave infra-red (MWIR and LWIR), and Passive MilliMeter Wave (PMMW) sensors. Flight demonstrations of the real-time system have been performed with both visible and LWIR image sensors. In the flight demonstrations, an LWIR image sensor was used for both day and night detection in both rural and urban settings with detection ranges in excess of 15,000'. The processing system is capable of processing image sizes up to 1024x1024 pixels at 30 frames per second.
\end{abstract}

Keywords: Wire Detection, Cable Detection, Passive Obstacle Detection, Wire Strike

\section{INTRODUCTION}

A key component of aircraft obstacle avoidance is the detection of power lines and cables. Within the general area of obstacle detection, power lines and cables present some unique detection challenges. Due to their small diameter (typically less than 1.25”) they do not show up in digital terrain maps and can be very difficult to detect visually, especially when seen against complex natural backgrounds. While radio towers, smoke stacks, cranes, and other tall, narrow man-made objects typically have warning lights to improve their visibility, most power lines and their supporting pylons and poles have no such visibility enhancements. In order to support a wide range of operating conditions, it is necessary to be able to detect power lines and cables during both day and night, and in reduced visibility conditions.

Required detection range is a function of aircraft speed and maneuverability. At $150 \mathrm{kt}$ (typical top speed for a helicopter) the aircraft covers $250 \mathrm{ft} / \mathrm{sec}$ while at a fixed wing speed of $250 \mathrm{kt}$ an aircraft covers $420 \mathrm{ft} / \mathrm{sec}$. If one uses a minimum reaction time of 3 seconds, then for a helicopter flying nap of the earth at full speed, the minimum useful detection range is $750 \mathrm{ft}$ while for a fixed wing aircraft it may be more like $1300 \mathrm{ft}$. A much more comfortable time lag of $10 \mathrm{sec}$ gives desired detection ranges of $2500 \mathrm{ft}$ and $4200 \mathrm{ft}$ respectively. High voltage power lines tend to be approximately 1 " or 1.25 " in diameter while local distribution lines are more typically $1 / 2$ " in diameter. However, the smaller distribution lines tend to be much closer to the ground and therefore pose much less of a flight hazard.

A number of different approaches are under development. These include sensors to detect electro-magnetic emissions from active power lines and various forms of lidar and radar. Boeing has been developing real-time passive wire detection systems since 1998, resulting in 1 patent and 2 published patent applications [1-3]. Passive wire detection has several distinct advantages over other approaches but also faces significant technical challenges. Unlike detectors of electro-magnetic emissions, both lidar/radar and passive detection techniques can detect both powered and un-powered lines and can provide precision location of the wires and cables. In comparison with active systems, the presence and operation of a passive system is completely undetectable. While lidar systems may be claimed to be Low Probability of Intercept / Low Probability of Detection (LPI/LPD), passive systems are by definition Zero Probability of Intercept / Zero Probability of Detection (ZPI/ZPD). Further, active systems are limited in range by their transmitter power and the cross section of the object to be detected.

Initial detection range, with zero false alarms, for the PODS wire detection system is 15,000 ft (36 sec at $250 \mathrm{kt}$ ). Detection strength increases to detection in over $50 \%$ of the image frames by $11,000 \mathrm{ft}$ ( $26 \mathrm{sec}$ at $250 \mathrm{kt}$ ) and continuous detection (100\% of frames) by $2300 \mathrm{ft}$ (5 sec at $250 \mathrm{kt})$.

*Boeing-SVS, Inc. 4411 The 25 Way NE, Albuquerque, NM 87109 
The biggest challenge for passive wire detection is that automatic real-time detection of wires in passive imagery is much more difficult than in 3D lidar data. In passive imagery, the wires are projected onto the background with a resulting loss of range information. This makes separation of wires from other background "wire like" objects such as roads, trees, ploughed fields, and so on much more difficult. Wires are typically not straight lines but instead catenary curves, they may be partially obscured by foreground objects, and they may appear at any orientation in the imagery. As a result, reliable detection of wires in passive imagery, without false alarms, has proven a daunting technical challenge. In this paper we present algorithms and results demonstrating that these obstacles have been overcome, resulting in a high performance passive wire detection system.

Both lidar systems and passive imaging systems operating in the visible and IR spectrum are degraded by presence of obscurants such as rain, fog, dust, or snow. In general, longer wavelengths tend to penetrate these obscurants better than shorter wavelengths. The PODS wire detection system can utilize any passive sensor but the current preferred embodiment is a Long Wave Infra-Red (LWIR) sensor operating in the 8-12 um waveband. This provides passive imaging in both day and night conditions and penetration of light to moderate dust, fog, and other obscurants. An LWIR sensor is also typically much smaller and lighter weight than an active lidar system, providing a significant benefit in an aircraft environment.

\section{SYSTEM DESCRIPTION}

\subsection{Algorithm}

\subsubsection{Requirements}

The passive wire detection algorithm must be capable of detecting wires and cables in passive imagery and of being implemented in real-time image processing hardware capable of handling reasonable image sizes. The image content which the algorithm must handle includes the ability to detect sub-pixel lines which may have either positive or negative contrast. The lines may be at any orientation in the imagery, and are typically curved (catenary). The lines may be partially obscured by foreground obstacles. In addition to single lines, the algorithm must detect groups of lines (bundles) having much greater cross-line extent than a single wire. The imagery may contain sharp edges (horizons, road edges, buildings), significant structure, and "wire like” objects such as distant, unresolved trees or roads.

The processing throughput requirement for the algorithm, when implemented in hardware is $1024 \times 1024$ pixel imagery at $30 \mathrm{~Hz}$. Further, since the algorithm is intended for real-time operation in flight, it cannot require manual adjustment of parameters. This means that it must be robust to changing perspectives due to motion of the aircraft. In particular, objects such as trees begin as highly unresolved, but as they are approached, become partially resolved and then highly resolved. This dynamic scene change poses a particular challenge for a robust, automatic algorithm.

\subsubsection{Algorithm Overview}

The current PODS wire detection algorithm consists of 4 processing stages listed below:

- Pre-processing to remove background clutter. This includes a Ring Median Filter to remove large scale clutter and a SUSAN (Smallest Univalue Segment Assimilating Nucleus) filter to remove high contrast "wire-like" clutter.

- Segment Detection to find wire like segments which in the next step will be linked together into lines. This step consists of a gradient phase operator and a vector kernel operator which together reject random noise and edges while preserving line segments.

- Segment Linker to link spatially separated line segments into larger scale lines and eliminate small isolated segment detections. This step consists of a bank of 16, 41x41 morphological filters implementing $\mathrm{M}$ out of $\mathrm{N}$ tests.

- Spatial \& Temporal Filter to eliminate temporary detections which are not persistent over multiple frames (reduces false alarm "flicker") and remove any remaining, persistent detections which are clearly not wire-like 
based on their spatial characteristics (length and width). The temporal filter is a decaying exponential recursive filter while the spatial filter clusters all adjacent pixels and rejects clusters smaller than a given size.

The PODS wire detection algorithm may be preceded by electronic image stabilization and a "bit picker" to reduce incoming imagery to 8 bits. The algorithm is summarized by the block diagram below:

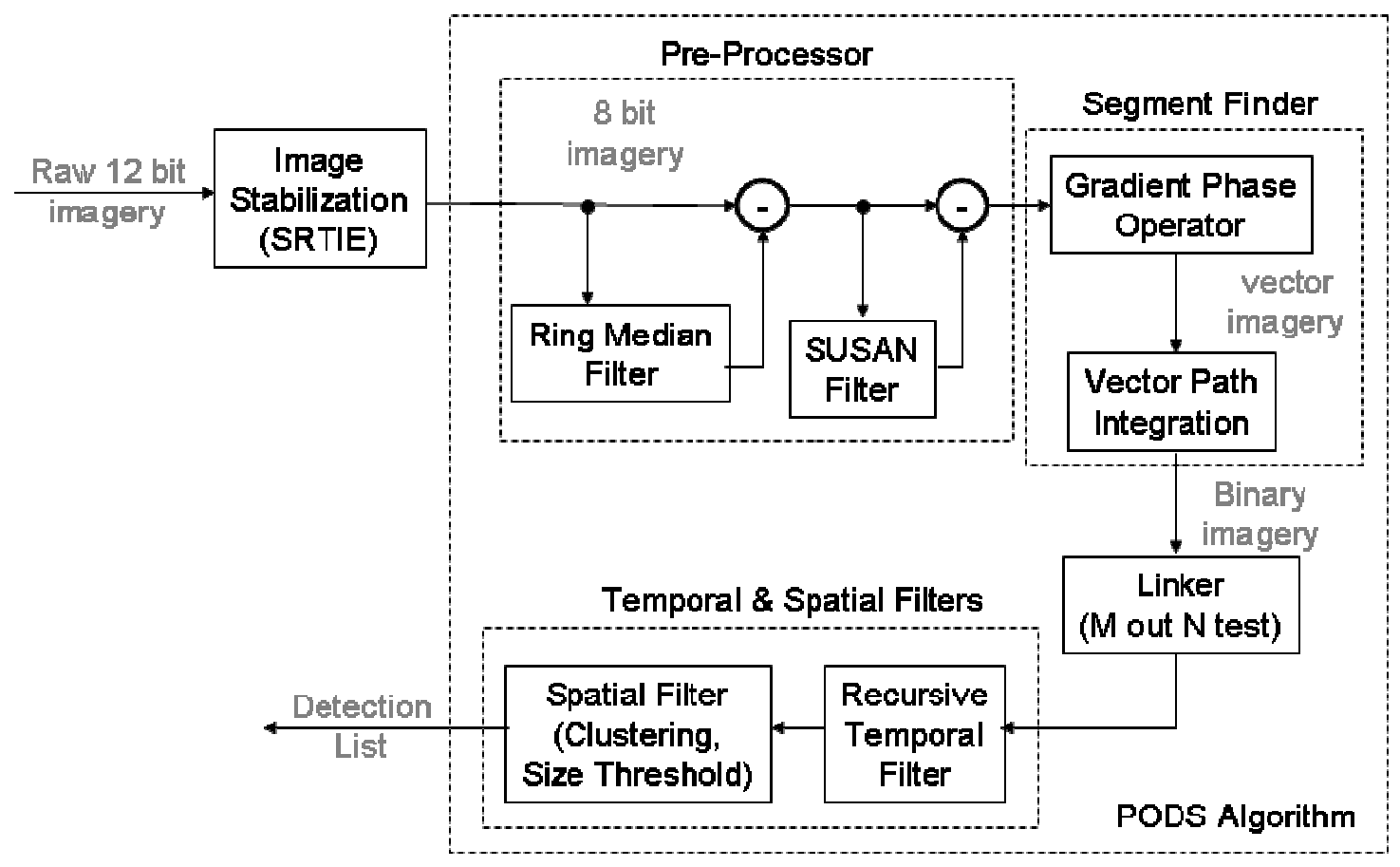

Block Diagram of PODS Algorithm

\subsubsection{Pre-Processing}

This consists of image processing functions to remove large scale and non wire-like clutter from the image. The 2 primary steps are a ring median filter to perform scale selection followed by a SUSAN filter to perform scale and contrast selection.

The ring median filter replaces each pixel with the median value of the pixels in a ring of radius " $r$ " from the pixel. This eliminates objects with a scale size less than the radius of the ring. We now subtract the resulting "ring median filter" image from the original image, leaving only objects with a scale size of less than " $\mathrm{r}$ ". This is typically wires, noise, and wire-like clutter. In order to allow bundles of closely spaced wires, the ring diameter must be large enough to pass not only single wires but also bundles of wires. However, this also allows additional clutter to pass. The Ring Median Filter is implemented as a $5 \times 5$ convolution kernel. The ring diameter is thus 5 pixels.

In order to separate true wires from wire-like clutter we recognize that wires typically exhibit lower contrast than clutter and hence we can utilize contrast to separate wires from other spatially similar clutter. A SUSAN filter (Smallest Univalue Segment Assimilating Nucleus) is applied to the output of the previous step. The SUSAN filter determines the mean value of all pixels in a $5 \times 5$ patch around each pixel which are within $\mathrm{N}$ grey scale values (above or below) of the center pixel and replaces the center pixel with this mean value. This eliminates low contrast features leaving an image with high contrast features intact and somewhat sharpened. We subtract this image from the original output of the ring median filter step to obtain an image in which we have removed the high contrast features but retained the low contrast features. 


\subsubsection{Segment Finder}

For each pixel the algorithm determines the degree to which its local surroundings are wire-like. The 2 primary steps in this stage are a gradient phase operator followed by vector sum kernel operator (essentially a vector path integration).

The gradient phase operator computes a vector which is orthogonal to the local intensity gradient at all pixels in the image scene. It uses 2, 5x5 convolution kernels, one for the $\mathrm{x}$ direction and one for the $\mathrm{y}$. The result along a line is shown pictorially below:

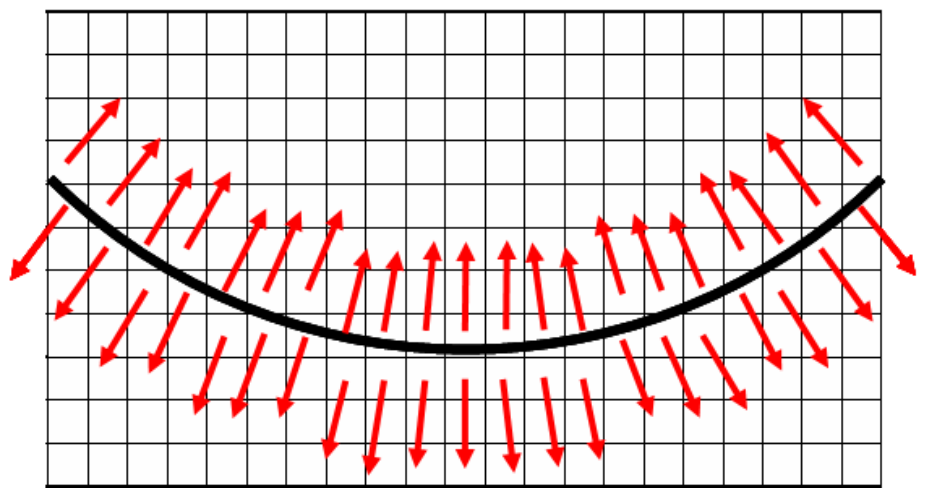

Result of gradient phase operator

The Vector sum kernel operator places a long thin kernel over each pixel perpendicular to the local phase. This would be along the wire if there is a wire in that pixel. The unit phasors (2D vectors) are weighted via the kernel and summed. The kernel multipliers on one side of the (hypothetical wire) are positive, while on the other side they are negative. If there is in fact a wire present, this will orient the phasors on opposite sides in the same direction so that they sum coherently. The vector sum (e.g. the kernel) may be represented mathematically thus:

$$
\rho_{1} e^{i \varphi_{1}}=\sum_{n=1}^{N} K_{n} e^{i \theta_{n}},
$$

where $K_{n}= \pm 1$ is the kernel multiplier for the $n^{\text {th }}$ non-zero kernel element ( $n=1$ for the central pixel), and $\theta_{n}$ is the phase for that element.

For random phase fields (no wires, no edges), $\left\langle\rho_{1}{ }^{2}\right\rangle=\mathrm{N}$ and $\varphi_{1}$ is uncorrelated with $\theta_{1}$. For perfectly aligned fields (edges), $\left\langle\rho_{1}{ }^{2}\right\rangle=0$ and $\varphi_{1}=\theta_{1}$. (modulo $\pi$ ). For a perfectly straight wire, $\left\langle\rho_{1}{ }^{2}\right\rangle=\mathrm{N}^{2}$ and $\varphi_{1}=\theta_{1}$. (modulo $\pi$ ). The value $\theta_{1}$ is the angle of the gradient for the pixel under evaluation. Examples of these cases are shown below: 
Random (White Noise)

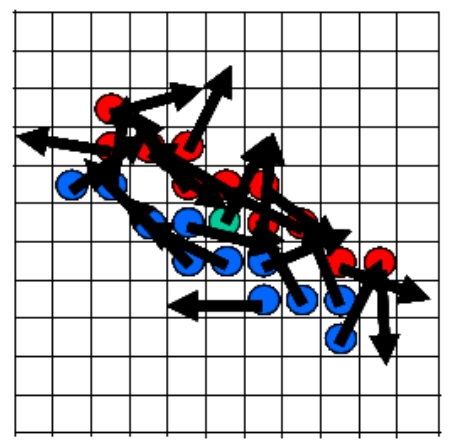

$\boldsymbol{K}_{n}=+\mathbf{1}$

$K_{n}=-1$

random pattern

$\sum_{n=1}^{N} K_{n} e^{i \theta_{n}}=4.66 e^{-1.08 i}=8 \times 1$

Small / Wrong Orientation $\Rightarrow$ Not a Wire Candidate
Uniform (Intensity Gradient)

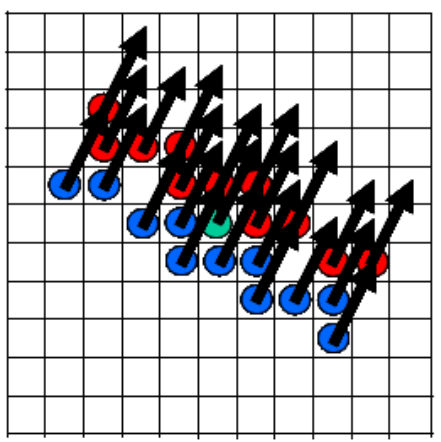

$\boldsymbol{K}_{n}=+1$

$\boldsymbol{K}_{n}=-1$

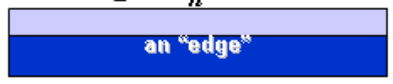

$=0 e^{1.05 i}=8 \mathrm{x}$

\section{Null / Correct Orientation} => Not a Wire Candidate
Wire

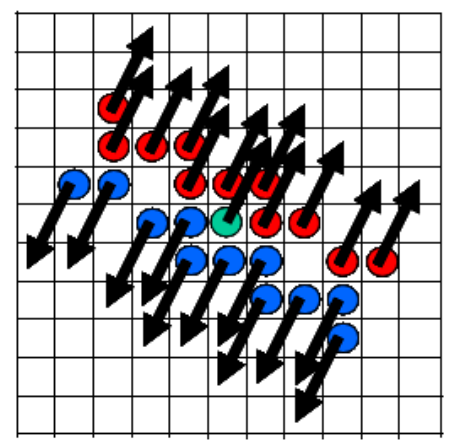

$K_{n}=+1$

$K_{n}=-1$

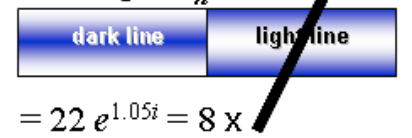

Large / Correct Orientation

$\Rightarrow$ Wire Candidate

Effect of the Vector Path Integration kernel operating on the gradient phase operator image. The pixel under evaluation is the central green pixel.

The size of the vector sum kernel is a critical algorithm performance parameter, driven by available computational resources. As the kernel size becomes larger, it becomes more effective at eliminating wire-like noise and edges while preserving and detecting the wires. Eventually, wire curvature places an upper limit on the useful size of the kernel and kernel sizes larger than this will begin to reject wires due to their catenary curve.

The output of this stage is a binary map of pixels which form accepted line segments. Pixels must both exceed the $\left\langle\rho_{1}{ }^{2}\right\rangle$ threshold and lie within the $\left|\varphi_{1}-\theta_{1}\right|$ threshold to be accepted.

\subsubsection{Segment Linker}

This stage examines spatially widely dispersed pixels to find large-scale wire-like structures. It utilizes a bank of 16 linear matched filters (41x41 kernel) implemented as $\mathrm{M}$ out of $\mathrm{N}$ tests. The purpose is to link significantly separated wire segments into larger scale segments. This step also rejects small isolated segment detections. The detected segment pixels are summed within each filter bank and if the total number of segment pixels (M) exceeds the threshold for that filter, the center pixel (the pixel under evaluation) is set to 1 (detection), otherwise it is set to 0 (no detection). Note that the threshold varies from filter to filter due to the differing lengths of the filter banks $(\mathrm{N})$, depending on angle. 


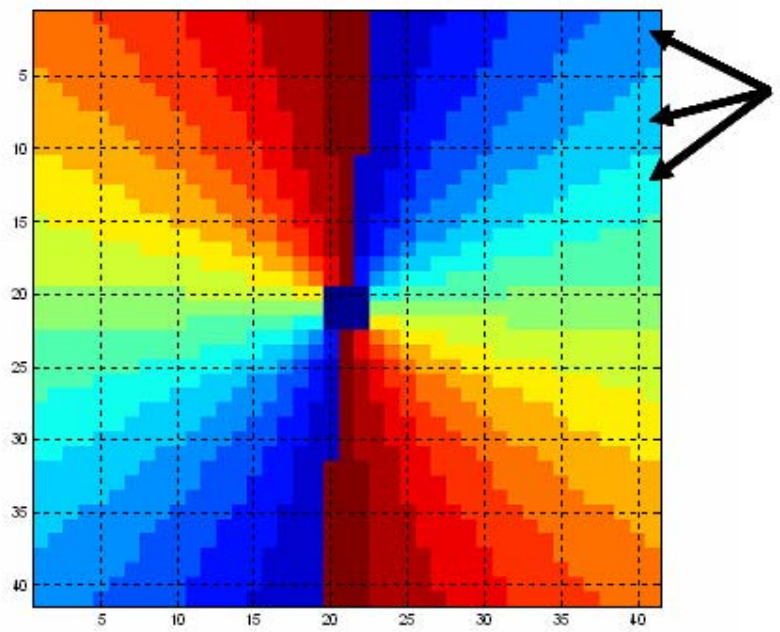

Each separate color in this $41 \times 41$ grid represents a separate filter bank.

\author{
If the number of wire candidate pixels \\ within any filter bank exceeds the \\ threshold for that filter bank, the linker \\ output corresponding to the center of \\ the $41 \times 41$ grid is set to "true."
}

Segment linker $41 \times 41 \mathrm{M}$ out $N$ filter bank for linking separated segments into larger lines

A single long wire segment can pass this filter bank, as can several short segments if they are all aligned with each other. However, isolated segments or segments with inconsistent orientations are rejected.

\title{
2.1.6 Temporal \& Spatial Filters
}

These image processing functions remove segment linker clutter and reinforce temporally persistent detections. An exponentially weighted, temporally recursive filter is used to eliminate single frame flickering false alarms. The spatial filter utilizes connected components and morphological processing to remove remaining persistent non-wire detections. The temporal filter is the only step in the processing chain which does not utilize single frame processing.

We use a recursive temporal filter to minimize the impact on latency. The filter may be written as:

$$
F_{N+1}=\alpha I_{N}+(1-\alpha) F_{N}
$$

where $\mathrm{I}_{\mathrm{N}}$ is the $\mathrm{N}^{\text {th }}$ input image, $\mathrm{F}_{\mathrm{N}}$ is the $\mathrm{N}^{\text {th }}$ filtered image, and $\alpha$ is an adjustable parameter. This operation can be made completely parallel in hardware, thus further reducing latency. This is a temporarily exponential decaying filter in which the sum of the image coefficients goes to unity for large N. Heuristically, $1 / \alpha$ is roughly the number of images with significant weighting in the filter $(0<\alpha<1)$. In each frame, all pixels below a threshold are discarded and of course new pixels from the incoming frame are added.

Following the temporal filter there may remain some persistent non wire-like objects, typically short and thick relative to persistent wire-like objects. A final "length-width" spatial filter is employed to eliminate these. We first perform a closure operation (a dilation followed by erosion) to close small gaps between pixel clusters. Dilation and erosion may be implemented in hardware as Rank Value Filters (RVF). Following the closure operation, a simple clustering is used and a threshold applied to eliminate small objects.

\subsection{Implementation}

\subsubsection{Processing Hardware}


The PODS real-time processing is implemented in a single 3U processing card. This MEVS (Modular Enhanced Vision System) card is a Boeing developed, standard image processing block used for the Boeing Enhanced and Synthetic Vision System (ESVS), Super-resolution Real-Time Image Enhancement (SRTIE) real-time image stabilization and super-resolution processing, and other high,-end real-time image processing applications. We interface the boards to external devices such as cameras and displays using fiber transition modules, such as RS-170 to fiber, cameraLink to fiber, and fiber to VGA or DVI. Multiple MEVS boards can be daisy chained using one of the fiber ports to pass data from board to board. A single MEVS board implementing the PODS algorithm is capable of processing 1024x1024 pixel imagery at 30 frames per second.

Due to the temporal filter in PODS, we find that the PODS performance is sensitive to image stabilization. Image stabilization can be performed mechanically using an active sensor Line Of Sight stabilization technique, or it can be performed using electronic image shifting techniques. We found that during the December 2007 flight test, with no image stabilization, we obtained detection ranges of approximately 1400'. When we post processed the imagery with electronic stabilization, we extended the detection range to over $7600 \mathrm{ft}$, a 5 times improvement. As a result, we have integrated a Boeing Super-resolution Real Time Image Enhancement (SRTIE) processor with PODS. The SRTIE is implemented in a MEVS board identical to the PODS MEVS board, so the complete system consists of a 2 MEVS boards. Imagery arrives at the SRTIE board via high speed serial fiber, the SRTIE performs real-time electronic image stabilization, and the resulting stabilized image is passed from the SRTIE processor to the PODS processor over one of the fiber ports. Output from LWIR camera is 12 bits, while the PODS algorithm is implemented as an 8 bit algorithm. The SRTIE processor includes a "bit picker" which selects the best 8 bits out of the 12 bit range to represent the imagery.

It should be noted that the SRTIE board implements real-time super-resolution (with latency due to the multi-frame nature of the super-resolution algorithm), image stabilization, and various image enhancement functions including dynamic grey scale optimization by region. Everything except the super-resolution imposes less than a frame of latency to the processing chain. For PODS, only the image stabilization and bit-picker are utilized.

\subsubsection{Sensors}

The PODS algorithm will work with any 2D imaging sensor and has been demonstrated using single frame imagery to work well with visible, MWIR, LWIR, and even Passive Millimeter Wave (PMMW) imagery. We have performed flight tests using both visible and LWIR sensors and obtained good results from each. However, LWIR sensors are the preferred sensor for PODS. They provide:

- $\quad$ Night time imagery not available from a visible band camera

- Equal image quality day or night

- Provides better contrast imagery than obtained from visible wavebands

- Improved penetration of dust, fog, and snow due to the longer wavelength (8-12 um)

It is important to note that metal power lines typically have a very low emissivity, so what is observed is the reflection of cold sky from the wire. This means that detection should work equally well with powered and un-powered lines.

The most critical requirement imposed on any sensor by the PODS algorithm is that the output be free of linear artifacts, since by design PODS is highly sensitive to these. In particular, interlace artifacts are not acceptable and a digital output, progressive scan camera is highly preferred. Further, imagery cannot go through image compression processing prior to input to the PODS since these algorithms often introduce subtle blocking artifacts which are detected by PODS.

For our flight tests, we selected an I2Tech uncooled micro-bolometer LWIR (8-12 um) camera (model HRU640) with a 9x7 degree FOV lens. The focal plane is a progressive scan at $30 \mathrm{~Hz}, 640 \times 480$ focal plane with pixels on a 25 um pitch. The NEdT is $<80 \mathrm{mK}$. The camera output is cameraLink which passes through a Boeing cameraLink to fiber transition module, providing fiber output to either the SRTIE processor or directly to the PODS. The 640x480 pixel focal plane together with the 9x7 degree FOV provides a single pixel IFOV of 250 urad. The camera weighs $1.5 \mathrm{lb}$, consumes less than $3 \mathrm{~W}$ power, and the dimensions are 6” L x 4" $\mathrm{H} \mathrm{x} 4$ ” W. 
A visible band Basler A202K camera having a 1004x1004 pixel progressive scan focal plane and a 26x26 degree FOV lens was also utilized. This provides an IFOV of 452 urad. The camera operated at $30 \mathrm{~Hz}$ with digital cameraLink output.

\subsubsection{Flight System}

The complete real-time system was demonstrated on a Boeing Little Bird (MD-530FF) helicopter in December, 2007, near Mesa, AZ. The system consisted of a chin mounted sensor pod, user displays and interface, the processor box, and a digital recording system to record both raw flight video as well as in-flight detection results. The sensor pod contained both the 1004x1004 visible band camera and the digital 640x480 LWIR camera. The sensor pod was hard mounted without vibration isolation. For the flight test we did not have the SRTIE real-time electronic image stabilization available.
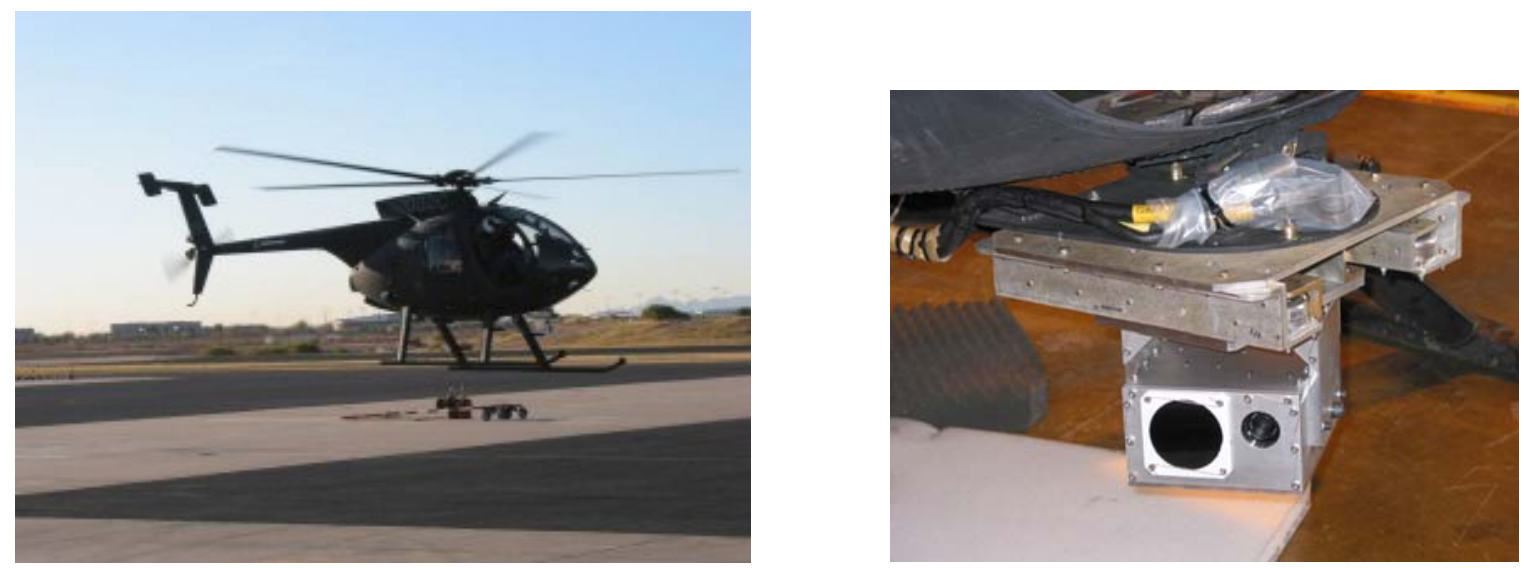

PODS flight test helicopter with chin mounted PODS sensor

The PODS can be utilized as a stand alone system consisting of sensor, processor, and user display, or it can be integrated into a larger system, such as the Boeing Enhanced and Synthetic Vision System [4].

\section{PERFORMANCE}

\subsection{Test Description}

During development of the PODS, flight data and still imagery was collected in various wavebands and with various backgrounds. This included imagery in the visible, Mid-Wave Infra-Red (MWIR, 3-5 um), Long Wave Infra-Red (LWIR, 8-12 um) and others. Imagery of various (angular) resolutions and pixel counts was collected. In addition still imagery of different scenes was obtained and utilized for algorithm evaluation. The best video sequences were from a fixed wing aircraft flight data collection in Albuquerque, NM using the 1004x1004 pixel visible band camera, and the December 2007 flight test on the Little Bird helicopter in Mesa, AZ using both the 1004x1004 pixel visible band camera and the 640x480 pixel LWIR camera.

The fixed wing flight data collection collected imagery of power lines with fields, plowed fields, roads, village structures, and horizon in the imagery. The flight geometry was generally perpendicular to the wires but sometimes on an angled approach.

The helicopter flight test took place mostly over desert terrain, using both rural and suburban terrain. Many structures were visible in the imagery, along with horizon, access roads, and saguaro cactus. Flight tests were performed with the LWIR sensor during both daytime and at night and during the daytime with the visible sensor. In order to adjust the realtime processing parameters, the sensor housing was pointed at an angle away from the direction of flight and the 
helicopter flew parallel to the wires for extended periods of time at constant offset distances. This provided time to adjust the parameters for best performance. Once this had been successfully performed at various distances, the sensor pod was pointed straight ahead and a perpendicular approach was flown beginning at a range of about 7600 feet and closing to less than 500 feet.

\subsection{Detection Results}

During the flight test we obtained good detections, day and night, to a range of about 1400 feet with the LWIR sensor and in fact obtained daytime detections with the LWIR sensor before the pilot detected the wires with his unaided eye. We also demonstrated, in flight, real-time processing and detection at $30 \mathrm{~Hz}$ using the 1004x1004 pixel visible band sensor, albeit with somewhat shorter range detections.

Analysis subsequent to the flight test indicated that the imagery exhibited a slow pitch oscillation which we believed degraded the performance of the temporal filter. As a result, we added the real-time image stabilization SRTIE card to provide stabilized imagery to the PODS processor and re-processed the imagery using real-time playback of our digital flight imagery. With the addition of image stabilization and a few other algorithm enhancements we found that we obtained detections with no false alarms, to the range limit (7600 feet) of our LWIR data. In order to further explore the limits of performance of the system, we performed a 2x2 binning on the flight data which, by halving the resolution, provided us with an approximation of flight data from twice the range of what we actually collected. Using this data we found that we were able to obtain initial, sporadic detections at ranges up to 15,000 ft in the LWIR video.

In order to examine performance as a function of range we adjusted the algorithm parameters so that we obtained absolutely no false alarms, not even a momentary single frame flicker. We then processed segments of the original LWIR imagery and the 2x2 binned imagery to determine the percent of frames in which detection occurred at various ranges. Note that this is NOT a Probability of Detection. Instead this is a measure of the strength of the detection: as range increases, the number of temporary drop-outs in detection increases. Initial detection is obtained at ranges greater than $15,000 \mathrm{ft}$ but these may be displayed as only intermittent flashes which might be missed if the user's attention is elsewhere. By the time the range has decreased to $11,000 \mathrm{ft}$ detection is obtained in approximately $50 \%$ of the image frames. At this point it would be hard for the user to avoid seeing the detections. By the time the range has decreased to $2300 \mathrm{ft}$ detection is continuous.

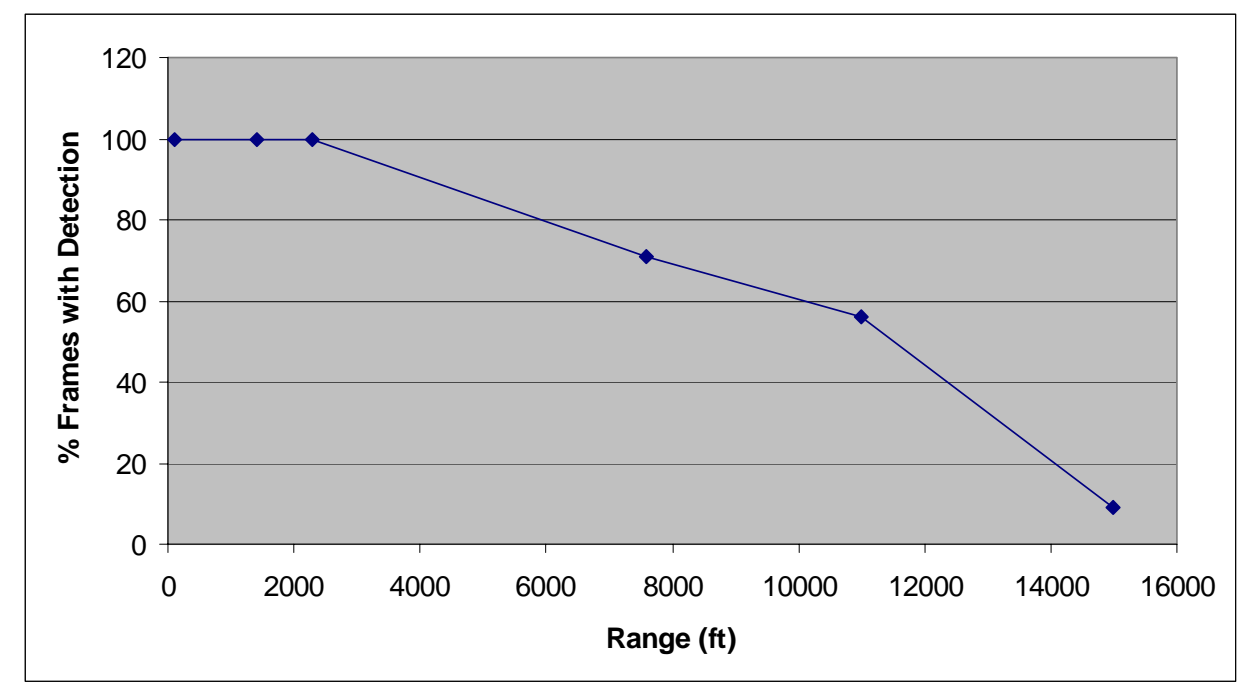

Strength of detection versus range with no false alarms: percent of frames showing detection.

Examples of detection results in the LWIR imagery corresponding to points on the graph are shown below. Mountains are clearly visible in the background. At the foot of the mountains is a suburban settlement including houses, roads, and 
other man made features. The imagery on the left is original imagery at a range of 7600 feet from the power lines while the imagery on the right has been $2 x 2$ binned to simulate a range of 15,000 ft. Red lines represent wire detections.
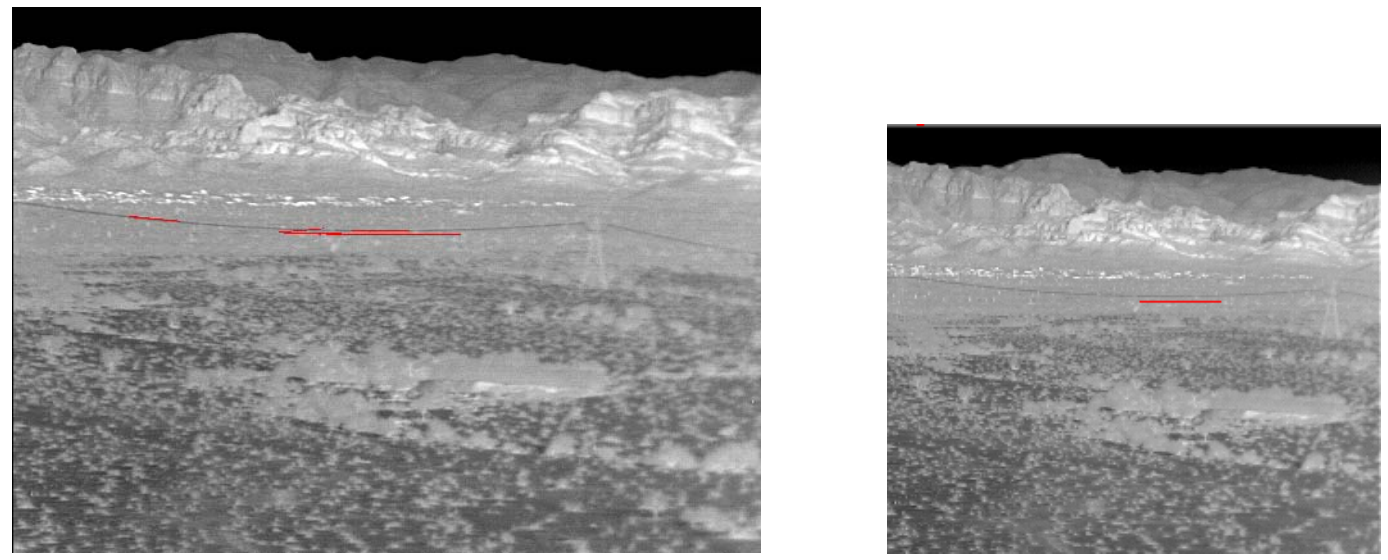

Wire detection with no false alarms in LWIR imagery. Left: Original imagery at a range of $7600 \mathrm{ft}$. Right: Imagery binned $2 \times 2$ to simulate 15,000 ft range

The observed detection ranges provide significant warning for wire hazards in the flight path. At 250 kts initial detection occurs around 35 seconds before impact, 50\% detection is obtained by 26 seconds, and continuous detection is obtained with 5 seconds remaining.

In addition to the video from the December 2007 flight test we also processed the visible band imagery from the fixed wing video collection as well as hand held single frame imagery. The example on the left below illustrates detection from the fixed wing flight video with plowed fields and structures in the background. The example on the right is a single still image which was processed for wire detection.
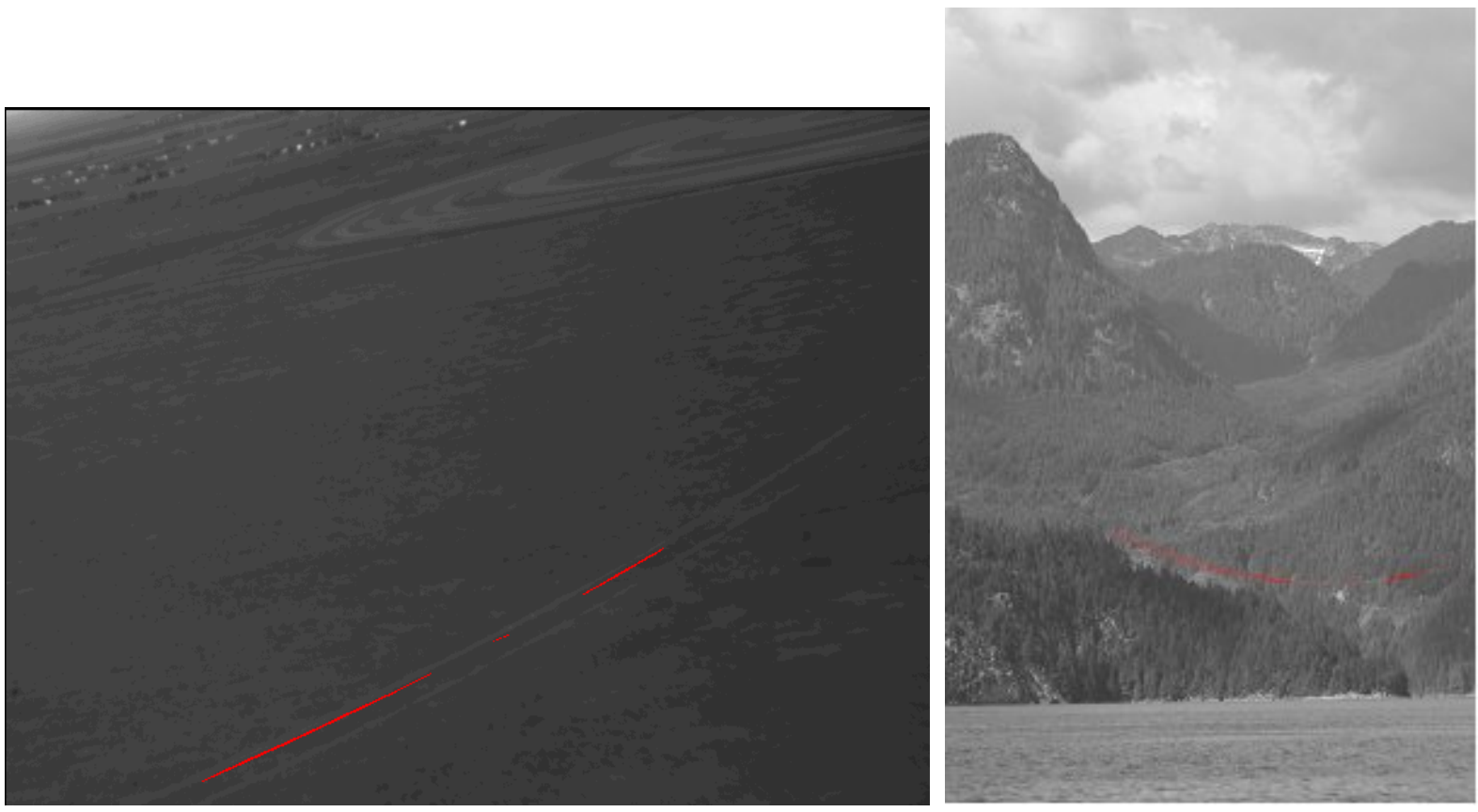

Visible band imagery wire detection. Left: video processing of flight imagery. Right: Still image 


\subsection{Detection range versus FOV}

The current system appears to significantly exceed detection range requirements. Therefore it is worthwhile investigating potential trade-offs in which we sacrifice detection range in order to obtain wider Field Of View (FOV) coverage. The current system has a 9x7 degree FOV aligned in the forward direction. There are two ways to increase the FOV, one is to increase the pixel count. The current LWIR sensor has a 640x480 pixel focal plane. If we maintain the same IFOV but increase the focal plane to $1024 \times 1024$, that will increase the FOV to $14 \times 14$ degrees. Since the IFOV remains unchanged we anticipate no change in performance.

Detection range is determined by contrast of the pixels containing wire with that of adjacent non-wire pixels. This is a function of the fundamental contrast of the wire with the scene background, reduced by the pixel fill factor of the wire. Since the wire is typically less than a pixel in diameter, a wire with a natural contrast of one, but only filling a quarter of a pixel will result in a contrast of only 0.25 . Following is a table showing the projected pixel size at various ranges for the 250 urad IFOV used in the current LWIR camera, and the resulting pixel fill factor for a 1" diameter wire.

\begin{tabular}{|c|c|c|}
\hline Range (ft) & Projected Pixel (in) & Fill Factor \\
\hline 1000 & 3.0 & 0.33 \\
\hline 2000 & 6.0 & 0.17 \\
\hline 4000 & 12.0 & 0.08 \\
\hline 6000 & 18.0 & 0.06 \\
\hline 7600 & 22.8 & 0.04 \\
\hline 15300 & 45.9 & 0.02 \\
\hline
\end{tabular}

Projected pixel size and pixel fill factor for a 1" diameter wire at various ranges for 250 urad IFOV pixels of the current LWIR sensor.

From this it appears that with zero false alarms, we are obtaining reliable (at least every 5 seconds) detection of wires with a fill factor of 0.02 .

If we assume that doubling the FOV will decrease the detection range by 50\% (because the pixel fill factor will decrease by 50\%), we can examine the expected detection performance as a function of FOV.

\begin{tabular}{|l|c|c|c|c|c|c|}
\hline FOV (deg) & $\mathbf{9 \times 7}$ & $\mathbf{3 2 \times 2 4}$ & $\mathbf{6 4 \times 4 8}$ & $\mathbf{1 4 \times 1 4}$ & $\mathbf{2 8 \times 2 8}$ & $\mathbf{5 6 \times 5 6}$ \\
\hline Sustained & 2300 & 657 & 329 & 2300 & 1150 & 575 \\
\hline $50 \%$ Frames & 11000 & 3142 & 1571 & 11000 & 5500 & 2750 \\
\hline Initial Detect. & 15300 & 4371 & 2186 & 15300 & 7650 & 3825 \\
\hline & \multicolumn{2}{|c|}{$640 \times 480$ pixel focal plane } & $1024 \times 1024$ pixel focal plane \\
\hline
\end{tabular}

Detection range (in ft) as a function of FOV for sustained, 50\%, and initial detection. The first three FOV columns are with the current 640x480 pixel LWIR focal plane while the last 3 columns are for a hypothetical 1024x1024 pixel focal plane with the same IFOV.

The 9x7 degree FOV is the current LWIR camera and observed performance. The 32x24 and 64x48 degree FOV values are for changing the 9x7 degree lens with different lenses we currently have for the same camera. The $14 \times 14$ degree FOV is for a hypothetical camera having a 1024x1024 pixel focal plane in which each pixel has the same 250 urad IFOV of the current $640 \times 480$ pixel camera. The 28x28 and 56x56 degree FOV columns correspond to simply doubling and quadrupling the FOV for the $1024 \times 1024$ pixel camera. These results indicate that a $1024 \times 1024$ pixel sensor with an approximately 30x30 degree FOV should give 18 seconds initial warning at $250 \mathrm{kt}$ and by 13 seconds the warning occurs in at least $50 \%$ of the image frames.

Additional algorithm enhancements have been identified and are available if needed to further enhance performance. 


\section{SUMMARY}

A passive wire detection system has been built and flight demonstrated. The flight imagery has also been re-processed on the ground, in real-time with additional enhancements to further improve performance. The following capabilities have been demonstrated:

- $\quad$ Real-time, in-flight processing and detection with $1004 x 1004$ pixel imagery at $30 \mathrm{~Hz}$

- Real-time, in-flight processing and detection of wires with both visible and LWIR cameras.

- Real-time, in flight processing and detection of wires at ranges up to 1400 feet, both day and night

- Real-time, in flight detection of wires during daytime before the pilot could see the wires with the unaided eye.

- Real-time post-processing initial detection of wires at 15,000 feet

- Real-time operation in 2, 3U processor cards: One for wire detection, one for image stabilization

- Demonstration of wire detection in visible, MWIR, LWIR, and other imagery

- Demonstration of wire detection with various backgrounds

The observed detection range provides at least 36 seconds warning for an aircraft flying at $250 \mathrm{kts}$. Since this is significantly greater than required for most applications, one can consider trading off range for increased Field Of View (FOV) coverage. Analysis suggests that useful detection ranges/times can be achieved with FOVs as large 30 degrees with the current algorithm. Additional algorithm improvements have been identified and can be easily implemented if required.

The results reported here represent first flight test results on a fairly limited range of data. Additional flight experience will be required to sustain these results and extend them to a broader range of terrain backgrounds and weather conditions. At the current time, parameter selection is performed manually and requires some skill and knowledge of the algorithm. Additional flight test experience is required for development of either a single, general purpose set of processing parameters, or an automatic scene dependent selection processing.

\section{ACKNOWLEDGEMENTS}

Many people beyond the authors contributed to this work. In particular, the authors would like to acknowledge the Boeing Little Bird helicopter flight crew including flight test engineer Mark Hardesty and test pilot David Guthrie-II.

\section{REFERENCES}

1. M.D. Nixon, R. Loveland, "Passive power line detection system for aircraft", US Patent, 6,940,994, Sept 6, 2005.

2. D.J. Yelton, R.L. Wright, "System and method for passive wire detection", US Patent application, 20070019838, Jan 25, 2007.

3. C.C. Witt, D.J. Yelton, J.C. Hansen, C.J. Musial, "System and method for passive wire detection", US Patent, 7,466,243, December 16, 2008.

4. J.N. Sanders-Reed, K. Bernier, J. Guell, "Enhanced \& Synthetic Vision System (ESVS) flight demonstration”, Proc SPIE, 6957, March 2008. 